\title{
GMR
}

\section{Molecular characterization, expression, and functional analysis of chicken TRAF6}

\author{
J. Jin ${ }^{1}$, J.S. Ran' ${ }^{1}$ C.W. Yang ${ }^{2}$, X.S. Jiang ${ }^{2}$, Y.G. Zhou ${ }^{1}$, Z.Q. Feng ${ }^{1}$, \\ Y. Wang', D. Lan' ${ }^{1}$, P. Ren ${ }^{1}$ and Y.P. Liu ${ }^{1}$ \\ ${ }^{1}$ Farm Animal Genetic Resources Exploration and Innovation Key Laboratory \\ of Sichuan Province, Sichuan Agricultural University, Chengdu Campus, \\ Chengdu, China \\ ${ }^{2}$ Sichuan Animal science academy, Chengdu, China \\ Corresponding author: Y.P. Liu \\ E-mail: liuyp578@yahoo.com \\ Genet. Mol. Res. 16 (1): gmr16019138 \\ Received September 2, 2016 \\ Accepted January 19, 2017 \\ Published February 8, 2017 \\ DOI http://dx.doi.org/10.4238/gmr16019138
}

Copyright $(2017$ The Authors. This is an open-access article distributed under the terms of the Creative Commons Attribution ShareAlike (CC BY-SA) 4.0 License.

\begin{abstract}
Tumor necrosis factor receptor-associated factor 6 (TRAF6) is a crucial adaptor molecule of the interleukin-1 receptor/ Toll-like receptor (IL-1/TLR) superfamily, which can trigger downstream signaling cascades involved in innate immunity. The function of TRAF6 has been clarified in mammals but is poorly understood in chicken. In our study, we investigated TRAF6 function in birds, particularly in chicken innate immune responses, by cloning and characterizing chicken TRAF6 (chTRAF6). The full-length coding sequence of chTRAF6 comprised $1638 \mathrm{bp}$ and encoded a 545-amino acid protein, which shares high sequence similarity with TRAF6 of other species and consists of four structurally conserved domains. Quantitative real-time polymerase chain reaction revealed that chTRAF6 was widely expressed in all tested tissues and its expression was induced in chicken embryo fibroblast cells treated with poly(I:C) and poly(dA:dT). Increased expression of chTRAF6 was observed both in vitro and in vivo following infection with Newcastle disease virus in
\end{abstract}


chickens. Taken together, these results suggest that chTRAF6 plays a vital role in host defense against viral infection in chicken.

Key words: Chicken; TRAF6; Innate immune system; Infection; mRNA expression

\section{INTRODUCTION}

Tumor necrosis factor receptor-associated factor (TRAF) family members (TRAF1-7) are intracellular signal transducers involved in inflammation and include immune receptor superfamilies, such as the tumor necrosis factor receptor (TNFR) superfamily and interleukin-1 receptor/Toll-like receptor (IL-1/TLR) family members (Inoue et al., 2000). All TRAFs have a similar protein structure, an N-terminal region, and C-terminal TRAF domain (Rothe et al., 1994). The N-terminal contains a RING-finger domain and a series of five $\mathrm{Zn}$-finger domains, which mediate protein ubiquitylation and activation of downstream signaling (Yang et al., 2015). The TRAF domain is comprised of a coiled-coil TRAF-N domain and a conserved TRAF-C domain, which is involved in self-association (Rothe et al., 1994) and interaction with various upstream regulators or downstream effectors (Zheng et al., 2010). TRAF6 was originally isolated by yeast two-hybrid screening using CD40 as bait (Ishida et al., 1996), and independently by screening of an expressed sequence tag (EST) library (Cao et al., 1996), and has been identified as a critical element for IL-1/TLR signaling. TRAF6 is the only TRAF family member that participates in both TNFR superfamily signaling pathways and TLR/IL1R superfamily pathways (Chung et al., 2002).

Previous studies have shown that TRAF6 regulates several signaling cascades involved in adaptive immunity, innate immunity, and bone metabolism (Ye et al., 2002). TRAF6 has a unique receptor specificity that does not overlap with that of other TRAF family members (Darnay et al., 1999). TRAF6 recognizes different binding sites of CD40 and receptor activator of nuclear factor $\mathrm{kB}$ (RANK), and other members of the TNFR superfamily (Pullen et al., 1998). TRAF6 does not directly bind to members of the IL-1R/TLR superfamily (Lomaga et al., 1999). Signaling proteins interleukin-1 receptor-associated kinase 1 (IRAK1) (Cao et al., 1996), interleukin-1 receptor-associated kinase 2 (IRAK2) (Muzio et al., 1997), interleukin-1 receptor-associated kinase M (IRAK-M) (Wesche et al., 1999), and possibly interleukin-1 receptor-associated kinase 4 (IRAK4) in the IL-1R/TLR pathway interact with TRAF6 (Suzuki et al., 2002). Members of the IRAK family are recruited to active receptors via interaction with adaptor proteins, such as myeloid differentiation factor 88 (Myd88), toll-interleukin 1 receptor domain containing adaptor protein (TIRAP), translocation associated membrane protein (TRAM), toll interacting protein (TOLLIP), and sterile alpha and HEAT/Armadillo motif containing (SARM) (Burns et al., 2000). Downstream signaling of TRAF6 is involved in K63-linked polyubiquitination (Deng et al., 2000). In addition, the retinoic acid-inducible gene I/melanoma differentiation-associated gene 5 (RIG-I/MDA5) signaling pathway requires the mitochondrial protein ISP-1 to form a signaling complex with TRAF6, which leads to the activation of TRAF6 and initiation of the innate immune response (West et al., 2011).

In recent years, the function and molecular mechanisms of TRAF6 action in the innate immune response have been intensively studied in mammals and yet remain unknown in chicken. Multiple studies have demonstrated that TRAF6 plays a crucial role in mammalian innate immunity (King et al., 2006). To investigate whether chTRAF6 plays an important role

Genetics and Molecular Research 16 (1): gmr16019138 
in innate immunity in chicken, we first cloned and characterized the cDNA of chTRAF6 and predicted its structure. Then, the expression of chTRAF6 was determined in all tissues tested. Finally, we analyzed the expression of chTRAF6 in vivo in chickens infected with Newcastle disease virus (NDV) and examined the expression of chTRAF6 in vitro in chicken embryo fibroblast cells (CEFs) stimulated with poly(I:C) or poly(dA:dT).

\section{MATERIAL AND METHODS}

\section{Chicken, tissues, cells, and viruses}

Specific pathogen-free (SPF) chickens are widely used experimental animals, and their use discounts interference by other pathogens. Forty White Leghorn SPF chickens from the house at the experiment farm of Sichuan Agricultural University (Sichuan, China) were employed for the experiment. Heart, liver, spleen, thymus, pancreas, small intestine, kidney, brain, pectoral muscle, and leg muscle were collected from three 14-day-old chickens for analysis of gene expression. All protocols used in this study were approved by Sichuan Agricultural University Animal Care and Use Committees. All tissues were snap-frozen in liquid nitrogen and stored at $-80^{\circ} \mathrm{C}$ until further use. $\mathrm{CEFs}$ were prepared from 9-day-old SPF chicken embryos and maintained in DEME (Life Technologies, USA) supplemented with $10 \%$ FBS (GIBCO, Life Technologies). The cells were cultured at $37^{\circ} \mathrm{C}$ with $5 \% \mathrm{CO}_{2} \mathrm{NDV}$ F48E9 and Mukteswar were obtained from the Department of Veterinary Medicine of Sichuan Agricultural University (Wenjiang, China).

\section{chTRAF6 gene cloning and bioinformatic analysis}

Primers (Table 1) were designed with Primer Premier 5.0 according to the published predicted sequence of chTRAF6 from Gallus gallus (GenBank accession No. XP_004941605.1), and used to amplify chTRAF6 cDNA by RT-PCR from spleen RNA. The amino acid sequences were blasted against the NCBI database using the BLAST program (http://blast.ncbi.nlm.nih. gov/) to determine sequence identities and deduce amino acid homology. Conserved domains of chTRAF6 were predicted using the SMART program (http://smart.embl-heidelberg.de/). Amino acid sequences were aligned using Clustal X2 (Figure 1) and a phylogenic tree (Figure 2) was constructed by MEGA 5.1 using 13 species (Table 2).

\begin{tabular}{|c|c|c|}
\hline Primer name & Primer sequence (5'- 3') & Application \\
\hline TRAF6-F & AGGACCAGGTTGTCTCCTGT & \multirow[t]{2}{*}{ Cloning } \\
\hline TRAF6-R & CCATCAAGTCCACAACACGG & \\
\hline GAPDH-F & AGGACCAGGTTGTCTGCTGT & \multirow[t]{2}{*}{ Real-time PCR } \\
\hline GAPDH-R & CCATCAAGTCGACAACACGG & \\
\hline TRAF6-F & ATGGAAGCCAAGCCAGAGTT & \multirow[t]{2}{*}{ Real-time PCR } \\
\hline TRAF6-R & ACAGCGCACCAGAAGGGTAT & \\
\hline
\end{tabular}

\section{NDV challenge and sample collection}

Thirty-six 2-week-old SPF chickens were randomly divided into three groups. Chickens in the first group were-infected intraperitoneally with NDV-F48E9, the second 
group was infected with NDV Mukteswar, and the third group was treated with phosphatebuffered saline as a control under the same conditions. At 1-, 2-, and 4-days post-infection, four chickens were euthanized from each group. Immune tissues were collected from all killed chicken, including the bursa of Fabricius, spleen, and thymus, snap frozen in liquid nitrogen, and then stored at $-80^{\circ} \mathrm{C}$. CEFs were collected at 4-, 6-, 8-, 12-, and 16-h post-infection with 1 multiplicity of infection of NDV F48E9 or Mukteswar, leaving uninfected cells as a control.

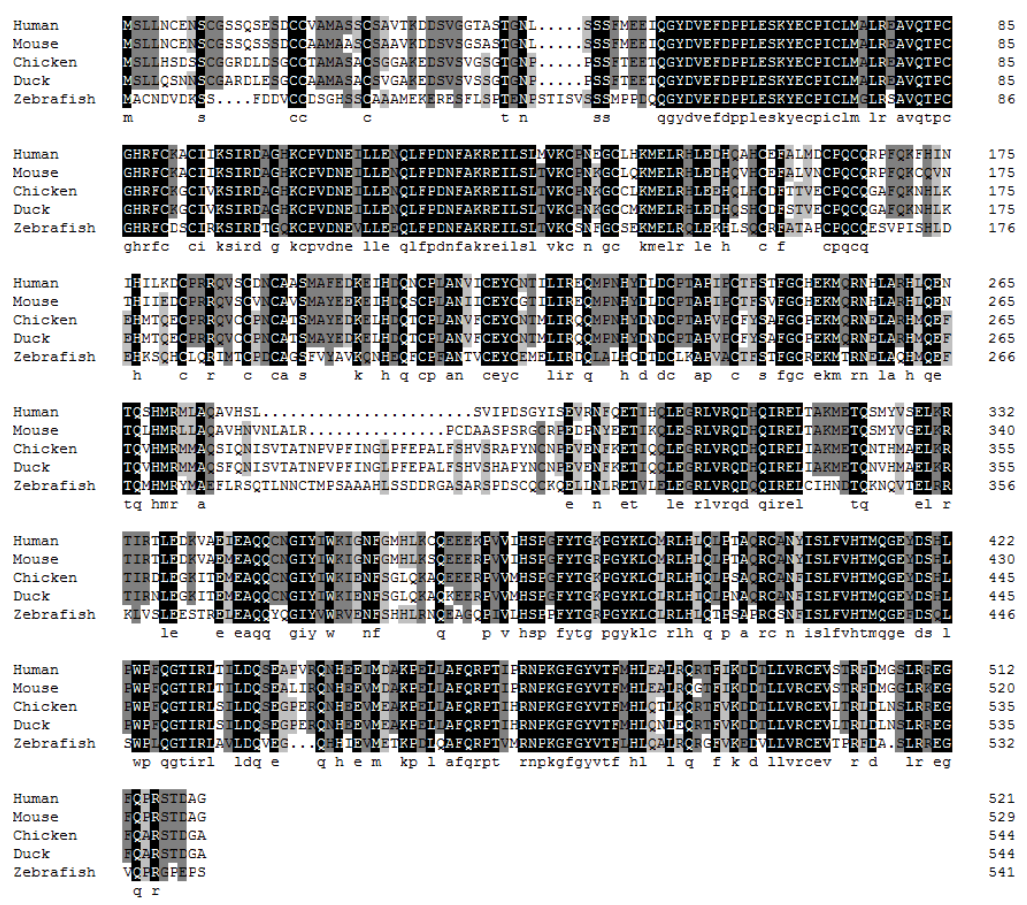

Figure 1. Alignment of human, mouse, chicken, duck, zebra fish TRAF6 amino acid sequences, performed using CLUSTAL X2. Letters below the alignments indicate identity, and gaps are marked with dashes.

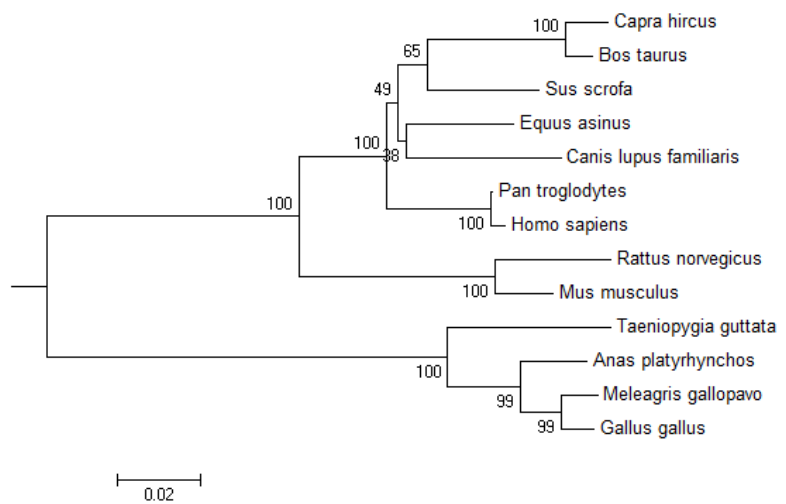

Figure 2. Phylogenetic analysis of the chTRAF6 protein. The tree was constructed using the MEGA 5.1 software and the scale bar is 0.02 .

Genetics and Molecular Research 16 (1): gmr16019138 
Table 2. GenBank accession No. of TRAF6 genes used in this study.

\begin{tabular}{l|c}
\hline Name of species & Accession No. \\
\hline Capra hircus & XP_005690148.1 \\
\hline Bos taurus & NP_001192515.1 \\
\hline Sus scrofa & XP_005652858.1 \\
\hline Equus asinus & XP_014706509.1 \\
\hline Canis lupus familiaris & XP_003432370.1 \\
\hline Pan troglodytes & XP_001154136.1 \\
\hline Homo sapiens & NP_004611.1 \\
\hline Rattus norvegicus & NP_001101224.1 \\
\hline Mus musculus & NP_033450.2 \\
\hline Taeniopygia guttata & XP_002198512.2 \\
\hline Anas platyrhynchos & XP_005011386.1 \\
\hline Meleagris gallopavo & XP_010709560.1 \\
\hline Gallus gallus & XP_004941605.1 \\
\hline
\end{tabular}

\section{Cell treatment with poly(I:C) and poly(dA:dT)}

To explore the effect of different stimuli on the expression of chTRAF6 in vitro, CEFs were first transfected using Lipofectamine 2000 (Invitrogen, USA), respectively, with poly(I:C) and poly(dA:dT) (InvivoGen, USA) according to the manufacturer protocol in a concentration-dependent manner, leaving uninfected cells as a control. Then, CEFs were treated with poly(I:C) and poly(dA:dT) in a time-dependent manner using the optimal concentration determined, respectively. Cells were harvested for total RNA extraction using TRIZOL reagent (Invitrogen) at the indicated time points.

\section{Total RNA extraction and cDNA synthesis}

Total RNA was extracted from tissues and CEFs with TRIZOL reagent (Invitrogen), according to the manufacturer protocol. Total RNA was incubated with RNase-free DNase I (OMEGA, USA) for $45 \mathrm{~min}$ at $37^{\circ} \mathrm{C}$. First-strand cDNA was synthesized from total RNA using the PrimeScript RT reagent Kit with a gDNA Eraser (TaKaRa, China) according to the manufacturer instruction.

\section{Quantitative real-time PCR}

Quantitative real-time PCR (qRT-PCR) was performed to determine the transcript level of chTRAF6. Each qRT-PCR mixture comprised $5 \mathrm{~mL}$ SYBR Green PCR master mix (TaKaRa), $3 \mathrm{~mL}$ nuclease-free water, $1 \mathrm{~mL} \mathrm{cDNA}$, and $0.5 \mathrm{~mL}$ each gene-specific primer $(10 \mathrm{mM}$; Table 1). The cycling protocol consisted of $3 \mathrm{~min}$ at $98^{\circ} \mathrm{C}$ for pre-incubation, then 40 cycles for 10 $\mathrm{s}$ at $98^{\circ} \mathrm{C}, 30 \mathrm{~s}$ at $60^{\circ} \mathrm{C}$, and $15 \mathrm{~s}$ at $72^{\circ} \mathrm{C}$. Relative gene expression was normalized using the endogenous control gene GAPDH and was calculated using the comparative $\mathrm{Ct}\left(2^{-\Delta \Delta \mathrm{Ct}}\right)$ method (Schmittgen and Livak, 2008). Melting curve analysis verified the specificity of the products.

\section{Statistical analysis}

All analyses were performed using SAS 9.3 (SAS Institute Inc., USA). The data were plotted and values are reported as means \pm standard error with GraphPad Prism 5 software (La Jolla, USA). P values $<0.05$ were considered significant.

Genetics and Molecular Research 16 (1): gmr16019138 


\section{RESULTS}

\section{Sequence analysis of $\operatorname{chTRAF6}$}

We amplified chTRAF6 cDNA by RT-PCR. The full-length coding sequence of chTRAF6 (Figure S1) was found to be $1638 \mathrm{bp}$ in length, and encoded a predicted 545 -amino acid residue protein. We predicted the structure of chTRAF6 using the SMART program (http://smart.embl-heidelberg.de), which indicated that chTRAF6 protein consisted of a RING finger domain (70-108 aa), two zinc fingers (204-261 aa), a coiled-coil region (314$371 \mathrm{aa}$ ), and a MATH domain (378-505 aa). Multiple sequence alignments revealed that chTRAF6 shares high sequence identity with TRAF6s from other species (Figure 1), but shares a relatively high level of identity with those of other birds (84-98\%), with the highest identity found with Meleagris gallopavo (98\%) and Anas platyrhynchos (96\%). Based on the deduced amino acid sequence of TRAF6 genes, a phylogenetic tree was constructed to identify their evolutionary relationships. As shown in Figure 2, TRAF6 genes in birds clustered in one group, which was divided into two clades. G. gallus was grouped into a cluster along with $M$. gallopavo and showed a particularly strong evolutionary relationship with Taeniopygia guttata and A. platyrhynchos.

\section{Tissue distribution of chTRAF6 mRNA}

The tissue distribution of chTRAF6 was determined by qRT-PCR. As shown in Figure 3, chTRAF6 was broadly expressed in all examined tissues with the highest expression observed in the lung, followed by the spleen, pancreas, thymus, small intestine, brain, kidney, and liver. Relatively low expression levels were observed in the brain and heart, and the lowest expression was found in the leg muscle.

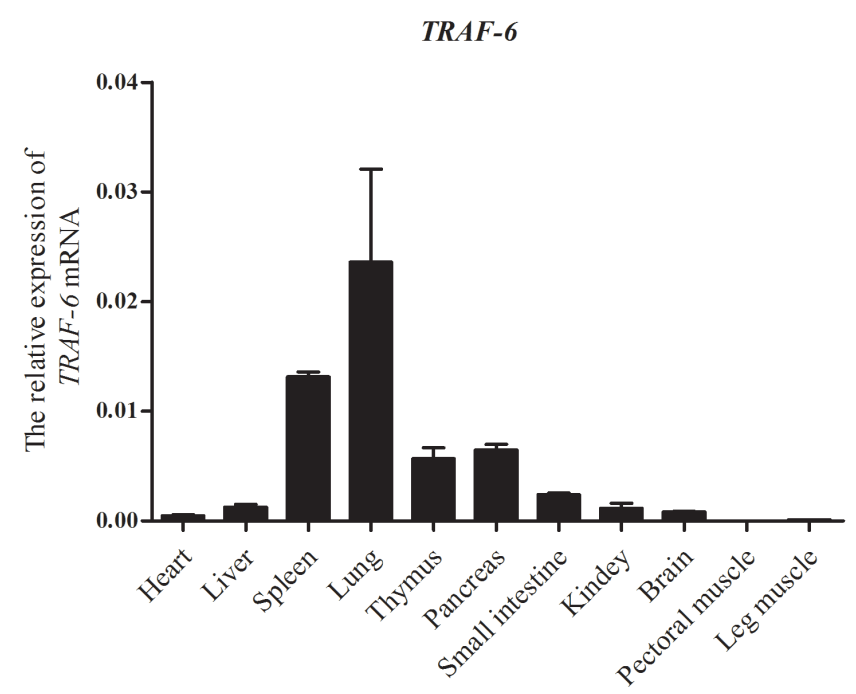

Figure 3. Tissue distribution of the $\operatorname{chTRAF6}$ gene in healthy chickens, as determined by qRT-PCR analysis. The values are reported as means $\pm \mathrm{SD}(\mathrm{N}=3)$.

Genetics and Molecular Research 16 (1): gmr16019138 


\section{Expression of chTRAF6 in immune organs of NDV-infected chickens}

We determined chTRAF6 expression levels in the immune organs of chickens following NDV F48E9 or Mukteswar infection to confirm whether chTRAF6 was also associated with the innate immune response in vivo. As shown in Figure 4, we observed the chTRAF6 gene to be significantly expressed in the bursa of Fabricius, spleen, and thymus of NDV-infected chickens.
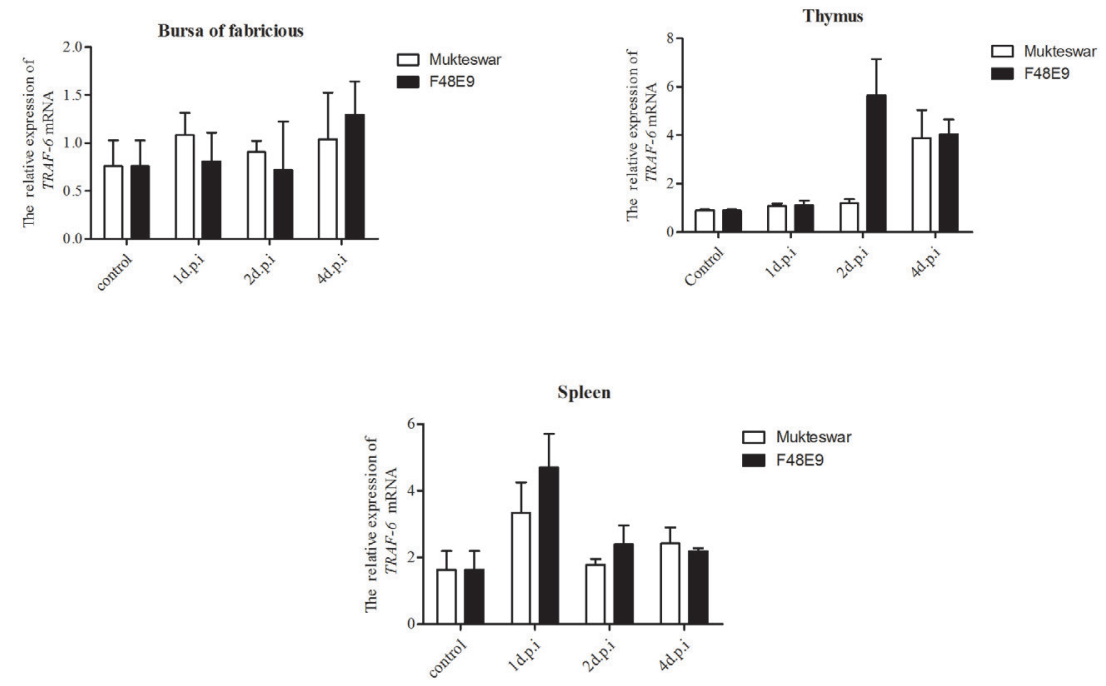

Figure 4. SPF chickens were infected with Newcastle disease virus (NDV), immune tissues including bursa of Fabricius, thymus and spleen were harvested at 1,2, and 4 days post-infection, respectively. The values are reported as means $\pm \mathrm{SD}(\mathrm{N}=3)$.

\section{Differential expression of $c h T R A F 6$ in NDV-infected CEFs}

We also explored the effect of NDV infection on chTRAF6 expression. Figure 5 shows that chTRAF6 expression levels in CEFs sharply increased from 6 to $16 \mathrm{~h}$ and peaked at 16 h. Meanwhile, chTRAF6 levels in Mukteswar-infected CEFs were significantly higher than in F48E9-infected CEFs.
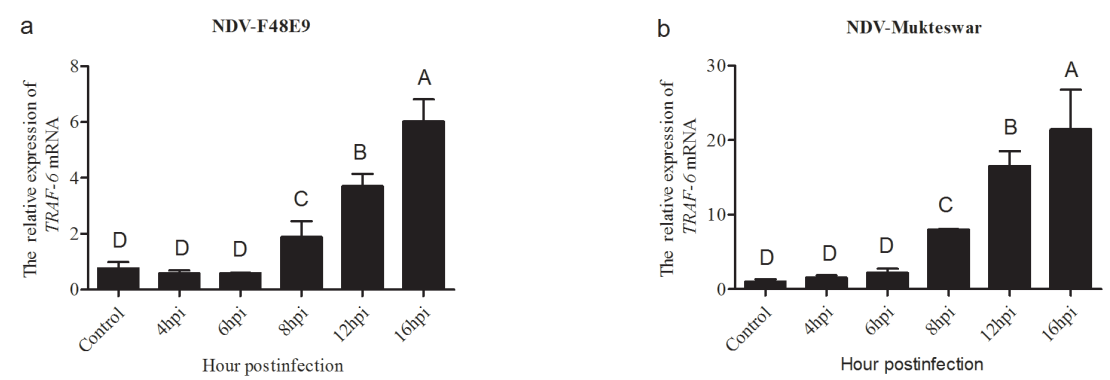

Figure 5. Expression of chTRAF6 in chicken embryo fibroblast cells (CEFs) following NDV infection. Cells were harvested at the specified time points. Different letters above bars indicate a significant difference $(\mathrm{P}<0.05)$. The values are reported as means $\pm \mathrm{SD}(\mathrm{N}=3)$.

Genetics and Molecular Research 16 (1): gmr16019138 


\section{Expression of $\operatorname{chTRAF6}$ in CEFs after poly(I:C) and poly(dA:dT) challenge}

We investigated whether the chTRAF6 gene was involved in the immune response of CEFs following infection with viral analogues. As shown in Figure 6a, the expression of chTRAF6 in CEFs significantly increased from control to $0.5 \mathrm{mg} / \mathrm{mL}$ poly(I:C), then decreased thereafter, reaching maximum expression at $0.5 \mathrm{mg} / \mathrm{mL}$ poly(I:C). An increase in the concentration of poly(dA:dT) tended to increase the expression of chTRAF6 in CEFs, which peaked following transfection with $4 \mathrm{mg} / \mathrm{mL}$ poly(dA:dT) (Figure $6 \mathrm{~b}$ ). Then, we examined the expression levels of chTRAF6 in CEFs subjected to poly(I:C) or poly(dA:dT) transfection at different time points using the optimum concentration, as described above. The expression levels of chTRAF6 were significantly decreased from 3 to $9 \mathrm{~h}$ in CEFs transfected with poly(I:C) $(0.5 \mathrm{mg} / \mathrm{mL})$, reaching maximum levels at 3-h post-transfection (Figure $6 \mathrm{c})$. Furthermore, the expression of chTRAF6 in CEFs gradually increased, beginning $3 \mathrm{~h}$ after transfection with poly $(\mathrm{dA}: \mathrm{dT})(4 \mathrm{mg} / \mathrm{mL})$ and peaking at $9 \mathrm{~h}$ (Figure $6 \mathrm{~d}$ ).
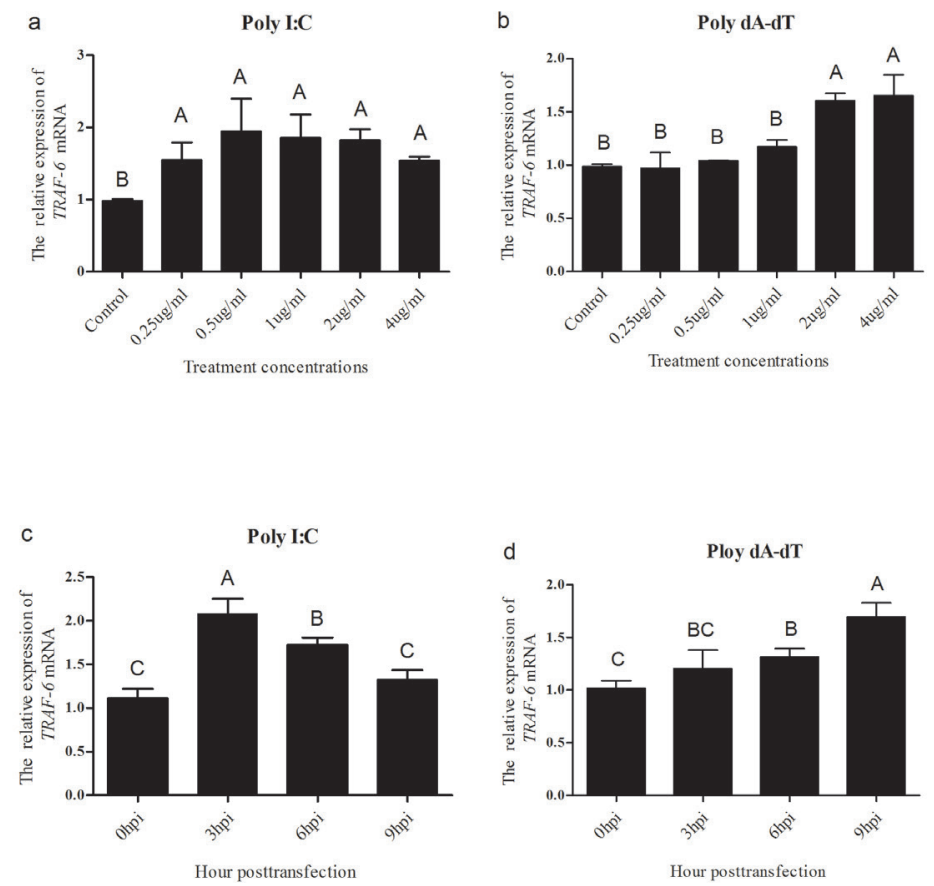

Figure 6. Expression of chTRAF6 in CEFs following poly(I:C) or poly(dA:dT) infection. a. b. Cells were treated with increasing concentrations of poly(I:C) or poly(dA:dT). c. d. Cells were harvested at the specified time points. Different letters above bars indicate significant difference $(\mathrm{P}<0.05)$. The values are reported as means $\pm \mathrm{SD}(\mathrm{N}=3)$.

\section{DISCUSSION}

TRAF6 is an adaptor molecule that functions as an E3 ubiquitin ligase and is essential for multiple signaling pathways, such as TNFR, TLR, and IL-1R (Poole et al., 2006). In this study, we cloned and identified the gene sequence of TRAF6 from chicken, which encoded a protein consisting of 545-amino acid residues. Results from BLAST analysis revealed the 
gene to have high nucleotide and amino acid sequence similarity to TRAF6 from other species, with the highest identity shared with G. gallus. In mammals, TRAF6 contains a RING finger, five zinc fingers, a coiled-coil region, and a MATH domain (Ishida et al., 1996). SMART analysis revealed that chTRAF6 possesses characteristics typical of TRAFs, with a RING finger domain, two zinc fingers of the TRAF-type, one coiled-coil region, and a MATH domain. There was high similarity between the gene sequence and the structural domains, suggesting chTRAF6 may have a function similar to that of other mammalian TRAF6s. The intact RING finger domain, together with the first zinc finger of TRAF6, is essential for auto-ubiquitination and interaction with Ubc13 (Lamothe et al., 2008). However, whether the reduced number of zinc fingers affects the function of chTRAF6 remains to be determined.

The phylogenetic tree based on the deduced amino acid sequences indicated that $G$. gallus TRAF6 was grouped into the bird cluster, and chTRAF6 showed a particularly strong evolutionary relationship with M. gallopavo TRAF6. Q-PCR analysis detected chTRAF6 in all tissues examined, indicating ubiquitous and constitutive expression of this gene. The most abundant chTRAF6 expression was found in the spleen, lung, thymus, and pancreas. In humans, the highest chTRAF6 expression was detected in the thymus, testis, and epidermis (Zapata et al., 2000). Previous studies suggested that TRAF6 is a crucial cytoplasmic adaptor molecule responsible for the pathogen-recognition receptors (PRR)-mediated innate immune response (Bradley and Pober, 2001). The tissue distribution of TRAF6 is an important determinant of its function, and can influence the capacity of cells to detect different microorganisms during different periods in different tissues (Cheng et al., 2015). The tissue distribution of TRAF6 has been previously examined in humans (Starczynowski et al., 2011), mice (Mason et al., 2004), and ducks (Zhai et al., 2015). Different species exhibit different tissue expression profiles of TRAF6; abundant expression of TRAF6 was observed in the spleen, which is the largest lymph organ, in humans, mice, and ducks, suggesting that this protein might be a critical factor for the immune system. chTRAF6 mRNA was highly expressed in immune-related tissue, including bursa of Fabricius, spleen, and thymus, indicating a functional TRAF6-mediated innate immune response to pathogenic challenges in chickens (Stockhammer et al., 2010).

NDV is a negative-strand RNA virus, which can cause great economic losses in the global fowl industry (Kapczynski et al., 2013). NDV is recognized primarily by RIG-I in human and murine cells (Kato et al., 2006). Although RIG-I is not found in chickens (Barber et al., 2010), infection with NDV can trigger the expression of type I IFNs, cytokines, and several IFN-stimulated genes in the spleen, macrophages, splenic leukocytes, and embryo fibroblasts (Rue et al., 2011), indicating the presence of an unknown viral sensor that compensate the functions of absence RIG-I.

In this study, we choose 2-week-old chickens to infect with NDV, because of their effective immune systems. We explored whether the chTRAF6 gene was expressed in CEFs and in immune organs of chickens following NDV infection. We demonstrated that TRAF6 expression significantly increased both in vivo and in vitro upon infection with NDV, suggesting an antiviral role for chTRAF6 in chickens. Taken together, these data indicate that chTRAF6 might function as a crucial protein in viral detection or in the activation of antiviral innate immune responses in chickens.

The innate system provides the first line of host defense against invading pathogens (Barbalat et al., 2011). Host cells express PRRs, which are engaged by pathogen-associated molecular patterns (PAMPs), thus triggering the innate immune response, leading to rapid production of type I IFNs (Takeuchi and Akira, 2010).

Genetics and Molecular Research 16 (1): gmr16019138 
Viral RNA and DNA can trigger immediate antiviral responses in human and mouse cells (Ablasser et al., 2009). Poly(dA:dT) does not favor the assembly or stability of nucleosomes in vitro. Poly(I:C) and poly(dA:dT) are synthetic analogs of viral nucleic acids, which trigger type I IFN responses in mammals (Gitlin et al., 2006), and dsRNA has long been considered a candidate viral PAMP. Treatment of mammalian cells with poly(I:C), which is a synthetic double-stranded RNA, induces type I IFN production via the activation of the transcription factors IRF-3 and NF-kB (Levy and Marié, 2004). Expression of TRAF6 in mammalian cells has been demonstrated in previous studies, showing that TRAF6 plays a crucial role in dsRNA-triggered antiviral signaling pathways (Mao et al., 2010). In our study, the expression of chTRAF6 was significantly induced in CEFs transfected with poly(I:C), which is recognized by MDA5 (Hayashi et al., 2014), suggesting that chTRAF6 is essential for the induction of effective innate immune responses. In addition, poly(dA:dT) is a repetitive synthetic double-stranded DNA, which is also indirectly detected by RIG-I (Thompson et al., 2011), and chTRAF6 expression was induced in CEFs following treatment with poly(dA:dT) in the present study. These results support the presence of potentially unknown DNA sensors in chicken cells that can detect poly(dA:dT) motifs. In addition, in contrast to poly(I:C), the expression levels of chTRAF6 followed a gradual upward trend in CEFs treated with poly(dA:dT). However, the mRNA and protein expression levels may exist in inverse proportions. To further verify the function of this gene, the expression levels of chTRAF6 should be quantified by western blotting. Taken together, these results indicate that infection with RNA and DNA viruses activates different antiviral pathways, and that chTRAF6 plays a crucial role in chicken defense against RNA and DNA viral infection.

\section{Conflicts of interest}

The authors declare no conflict of interest.

\section{ACKNOWLEDGMENTS}

Research supported by the Open Fund of Farm Animal Genetic Resources Exploration and Innovation Key Laboratory of Sichuan Province (Grant \#2016NYZ0043).

\section{REFERENCES}

Ablasser A, Bauernfeind F, Hartmann G, Latz E, et al. (2009). RIG-I-dependent sensing of poly(dA:dT) through the induction of an RNA polymerase III-transcribed RNA intermediate. Nat. Immunol. 10: 1065-1072. http://dx.doi. org/10.1038/ni.1779

Barbalat R, Ewald SE, Mouchess ML and Barton GM (2011). Nucleic acid recognition by the innate immune system. Annu. Rev. Immunol. 29: 185-214. http://dx.doi.org/10.1146/annurev-immunol-031210-101340

Barber MR, Aldridge JR, Jr., Webster RG and Magor KE (2010). Association of RIG-I with innate immunity of ducks to influenza. Proc. Natl. Acad. Sci. USA 107: 5913-5918.http://dx.doi.org/10.1073/pnas.1001755107

Bradley JR and Pober JS (2001). Tumor necrosis factor receptor-associated factors (TRAFs). Oncogene 20: 6482-6491. http://dx.doi.org/10.1038/sj.onc. 1204788

Burns K, Clatworthy J, Martin L, Martinon F, et al. (2000). Tollip, a new component of the IL-1RI pathway, links IRAK to the IL-1 receptor. Nat. Cell Biol. 2: 346-351. http://dx.doi.org/10.1038/35014038

Cao Z, Henzel WJ and Gao X (1996). IRAK: a kinase associated with the interleukin-1 receptor. Science 271: 1128-1131. http://dx.doi.org/10.1126/science.271.5252.1128

Cheng Y, Sun Y, Wang H, Yan Y, et al. (2015). Chicken STING mediates activation of the IFN gene independently of the RIG-I gene. J. Immunol. 195: 3922-3936. http://dx.doi.org/10.4049/jimmunol.1500638

Genetics and Molecular Research 16 (1): gmr16019138 
Chung JY, Park YC, Ye H and Wu H (2002). All TRAFs are not created equal: common and distinct molecular mechanisms of TRAF-mediated signal transduction. J. Cell Sci. 115: 679-688.

Darnay BG, Ni J, Moore PA and Aggarwal BB (1999). Activation of NF-kappaB by RANK requires tumor necrosis factor receptor-associated factor (TRAF) 6 and NF-kappaB-inducing kinase. Identification of a novel TRAF6 interaction motif. J. Biol. Chem. 274: 7724-7731. http://dx.doi.org/10.1074/jbc.274.12.7724

Deng L, Wang C, Spencer E, Yang L, et al. (2000). Activation of the IkappaB kinase complex by TRAF6 requires a dimeric ubiquitin-conjugating enzyme complex and a unique polyubiquitin chain. Cell 103: 351-361. http://dx.doi. org/10.1016/S0092-8674(00)00126-4

Gitlin L, Barchet W, Gilfillan S, Cella M, et al. (2006). Essential role of mda-5 in type I IFN responses to polyriboinosini c:polyribocytidylic acid and encephalomyocarditis picornavirus. Proc. Natl. Acad. Sci. USA 103: 8459-8464. http:// dx.doi.org/10.1073/pnas.0603082103

Hayashi T, Watanabe C, Suzuki Y, Tanikawa T, et al. (2014). Chicken MDA5 senses short double-stranded RNA with implications for antiviral response against avian influenza viruses in chicken. J. Innate Immun. 6: 58-71. http://dx.doi. org/10.1159/000351583

Inoue Ji, Ishida T, Tsukamoto N, Kobayashi N, et al. (2000). Tumor necrosis factor receptor-associated factor (TRAF) family: adapter proteins that mediate cytokine signaling. Exp. Cell Res. 254: 14-24. http://dx.doi.org/10.1006/ excr.1999.4733

Ishida T, Mizushima Si, Azuma S, Kobayashi N, et al. (1996). Identification of TRAF6, a novel tumor necrosis factor receptor-associated factor protein that mediates signaling from an amino-terminal domain of the CD40 cytoplasmic region. J. Biol. Chem. 271: 28745-28748. http://dx.doi.org/10.1074/jbc.271.46.28745

Kapczynski DR, Afonso CL and Miller PJ (2013). Immune responses of poultry to Newcastle disease virus. Dev. Comp. Immunol. 41: 447-453. http://dx.doi.org/10.1016/j.dci.2013.04.012

Kato H, Takeuchi O, Sato S, Yoneyama M, et al. (2006). Differential roles of MDA5 and RIG-I helicases in the recognition of RNA viruses. Nature 441: 101-105. http://dx.doi.org/10.1038/nature04734

King CG, Kobayashi T, Cejas PJ, Kim T, et al. (2006). TRAF6 is a T cell-intrinsic negative regulator required for the maintenance of immune homeostasis. Nat. Med. 12: 1088-1092.http://dx.doi.org/10.1038/nm1449

Lamothe B, Campos AD, Webster WK, Gopinathan A, et al. (2008). The RING domain and first zinc finger of TRAF6 coordinate signaling by interleukin-1, lipopolysaccharide, and RANKL. J. Biol. Chem. 283: 24871-24880. http:// dx.doi.org/10.1074/jbc.M802749200

Levy DE and Marié IJ (2004). RIGging an antiviral defense - it's in the CARDs. Nat. Immunol. 5: 699-701.http://dx.doi. org/10.1038/ni0704-699

Lomaga MA, Yeh WC, Sarosi I, Duncan GS, et al. (1999). TRAF6 deficiency results in osteopetrosis and defective interleukin-1, CD40, and LPS signaling. Genes Dev. 13: 1015-1024.http://dx.doi.org/10.1101/gad.13.8.1015

Mao AP, Li S, Zhong B, Li Y, et al. (2010). Virus-triggered ubiquitination of TRAF3/6 by cIAP1/2 is essential for induction of interferon-beta (IFN-beta) and cellular antiviral response. J. Biol. Chem. 285: 9470-9476. http://dx.doi. org/10.1074/jbc.M109.071043

Mason NJ, Fiore J, Kobayashi T, Masek KS, et al. (2004). TRAF6-dependent mitogen-activated protein kinase activation differentially regulates the production of interleukin-12 by macrophages in response to Toxoplasma gondii. Infect. Immun. 72: 5662-5667.http://dx.doi.org/10.1128/IAI.72.10.5662-5667.2004

Muzio M, Ni J, Feng P and Dixit VM (1997). IRAK (Pelle) family member IRAK-2 and MyD88 as proximal mediators of IL-1 signaling. Science 278: 1612-1615. http://dx.doi.org/10.1126/science.278.5343.1612

Poole E, King CA, Sinclair JH and Alcami A (2006). The UL144 gene product of human cytomegalovirus activates NFkappaB via a TRAF6-dependent mechanism. EMBOJ. 25:4390-4399.http://dx.doi.org/10.1038/sj.emboj.7601287

Pullen SS, Miller HG, Everdeen DS, Dang TT, et al. (1998). CD40-tumor necrosis factor receptor-associated factor (TRAF) interactions: regulation of CD40 signaling through multiple TRAF binding sites and TRAF hetero-oligomerization. Biochemistry 37: 11836-11845.http://dx.doi.org/10.1021/bi981067q

Rothe M, Wong SC, Henzel WJ and Goeddel DV (1994). A novel family of putative signal transducers associated with the cytoplasmic domain of the $75 \mathrm{kDa}$ tumor necrosis factor receptor. Cell 78: 681-692. http://dx.doi.org/10.1016/0092$\underline{8674(94) 90532-0}$

Rue CA, Susta L, Cornax I, Brown CC, et al. (2011). Virulent Newcastle disease virus elicits a strong innate immune response in chickens. J. Gen. Virol. 92: 931-939. http://dx.doi.org/10.1099/vir.0.025486-0

Schmittgen TD and Livak KJ (2008). Analyzing real-time PCR data by the comparative C(T) method. Nat. Protoc. 3: 1101-1108. http://dx.doi.org/10.1038/nprot.2008.73

Starczynowski DT, Lockwood WW, Deléhouzée S, Chari R, et al. (2011). TRAF6 is an amplified oncogene bridging the RAS and NF-kB pathways in human lung cancer. J. Clin. Invest. 121: 4095-4105.http://dx.doi.org/10.1172/JCI58818

Stockhammer OW, Rauwerda H, Wittink FR, Breit TM, et al. (2010). Transcriptome analysis of Traf6 function in the innate

Genetics and Molecular Research 16 (1): gmr16019138 
immune response of zebrafish embryos. Mol. Immunol. 48: 179-190.http://dx.doi.org/10.1016/j.molimm.2010.08.011 Suzuki N, Suzuki S, Duncan GS, Millar DG, et al. (2002). Severe impairment of interleukin-1 and Toll-like receptor signalling in mice lacking IRAK-4. Nature 416: 750-756. http://dx.doi.org/10.1038/nature736

Takeuchi O and Akira S (2010). Pattern recognition receptors and inflammation. Cell 140: 805-820. http://dx.doi. org/10.1016/j.cell.2010.01.022

Thompson MR, Kaminski JJ, Kurt-Jones EA and Fitzgerald KA (2011). Pattern recognition receptors and the innate immune response to viral infection. Viruses 3: 920-940. http://dx.doi.org/10.3390/v3060920

Wesche H, Gao X, Li X, Kirschning CJ, et al. (1999). IRAK-M is a novel member of the Pelle/interleukin-1 receptorassociated kinase (IRAK) family. J. Biol. Chem. 274: 19403-19410. http://dx.doi.org/10.1074/jbc.274.27.19403

West AP, Shadel GS and Ghosh S (2011). Mitochondria in innate immune responses. Nat. Rev. Immunol. 11: 389-402. http://dx.doi.org/10.1038/nri2975

Yang HL, Feng ZQ, Zeng SQ, Li SM, et al. (2015). Molecular cloning and expression analysis of TRAF3 in chicken. Genet. Mol. Res. 14: 4408-4419. http://dx.doi.org/10.4238/2015.April.30.14

Ye H, Arron JR, Lamothe B, Cirilli M, et al. (2002). Distinct molecular mechanism for initiating TRAF6 signalling. Nature 418: 443-447.http://dx.doi.org/10.1038/nature00888

Zapata JM, Krajewska M, Krajewski S, Kitada S, et al. (2000). TNFR-associated factor family protein expression in normal tissues and lymphoid malignancies. J. Immunol. 165: 5084-5096. http://dx.doi.org/10.4049/jimmunol.165.9.5084

Zhai Y, Luo F, Chen Y, Zhou S, et al. (2015). Molecular characterization and functional analysis of duck TRAF6. Dev. Comp. Immunol. 49: 1-6. http://dx.doi.org/10.1016/j.dci.2014.11.006

Zheng C, Kabaleeswaran V, Wang Y, Cheng G, et al. (2010). Crystal structures of the TRAF2: cIAP2 and the TRAF1: TRAF2: cIAP2 complexes: affinity, specificity, and regulation. Mol. Cell 38: 101-113. http://dx.doi.org/10.1016/j. $\underline{\text { molcel.2010.03.009 }}$

\section{Supplementary material}

Figure S1. Nucleotide and deduced amino acid sequences of chTRAF6. The stop codon is indicated by an asterisk.

Genetics and Molecular Research 16 (1): gmr16019138 